\title{
CdTe quantum dots precipitation of monodisperse fractions from colloid solutions
}

\author{
O.A. Savchuk ${ }^{1}$, L.I. Trishchuk ${ }^{1}$, I.A. Mazarchuk ${ }^{1}$, V.M. Tomashik ${ }^{1}$, Z.F. Tomashik ${ }^{1}$, \\ O.P. Dimitriev', S.D. Boruk ${ }^{2}$, D.O. Kapush ${ }^{3}$ \\ ${ }^{I} V$. Lashkaryov Institute of Semiconductor Physics, NAS of Ukraine, \\ 41, prospect Nauky,03028 Kyiv, Ukraine; e-mail: savchuk-olja@rambler.ru \\ ${ }^{2}$ Yu. Fedkovych Chernivtsi National University, 2, Kotsubinsky str., 58012 Chernivtsi, Ukraine \\ ${ }^{3}$ I. Frantsevich Institute for Problems of Materials Science, NAS of Ukraine, \\ 3, Krzhizhanivsky str., 03680 Kyiv-142, Ukraine
}

\begin{abstract}
CdTe nanocrystals were prepared in aqueous solution by the reaction between $\mathrm{Cd}^{2+}$ and $\mathrm{H}_{2} \mathrm{Te}$, obtained electrochemically in a galvanostatic cell, in the presence of thioglycolic acid. Subsequently, we have investigated precipitation of monodisperse fractions of CdTe quantum dots from polydisperse colloid solutions. In addition, the photoluminescence characteristics of these systems were studied in detail.
\end{abstract}

Keywords: cadmium compounds, quantum dots, nanocrystals, photoluminescence spectra.

Manuscript received 23.07.10; accepted for publication 02.12.10; published online 30.12.10.

\section{Introduction}

The modern methods of obtaining CdTe nanocrystals (NCs) are widely used in a number of fields of science and engineering because of detection of quantum size effects [1-3]. The physical methods for obtaining them provide a high degree of parameter control in a system, but they are complicated and need bulky and expensive equipments. Contrary, the chemical methods, for example colloid synthesis, are simpler. The latter is based on usage of stabilizers for prevention of the aggregative process. Stabilizers should form a strong bond with surface of NCs and have a high affinity with solvent.

The quantum dots (QDs) of cadmium chalcogenides attract the great interest of researchers. These chalcogenides are promising materials for microelectronics of a new type because of detection of quantum size effects. These effects are observed more clearly in CdTe NCs than others $\mathrm{CdB}^{\mathrm{VI}}$, where $\mathrm{B}^{\mathrm{VI}}-\mathrm{S}$ and $\mathrm{Se}$ because of large exciton Bohr radius ( $a_{w}=7.5 \mathrm{~nm}$; for example, for CdSe $a_{w}=5.4 \mathrm{~nm}$ [4]). Nevertheless, CdTe are the least investigated among nanodimensional cadmium chalcogenides. This is due to the complicated obtaining its colloid solutions caused by decomposition of $\mathrm{Te}$ (II) compounds and their high toxicity $[5,6]$.
The colloid QDs are characterized by a relatively narrow size distribution. On the other hand, when measuring the ensembles of these QDs their emission spectra have a full width at half maximum of a few $\mathrm{meV}$. Probably, the wide region of emission energy is related with the residual size distribution or this is a property of the colloid QDs. The majority of researches incline to the latter. In spite of a full width at half maximum of individual colloid QD is less than $0.1 \mathrm{meV}$, its emission spectrum shifts with the lapse of time accidentally. Such characteristic is named the spectral diffusion or spectral leap. For the first time, it was observed at cryogenic temperatures and, subsequently, at room temperature. Probably, the spectral diffusion is associated with local surroundings of QDs which form a high fluctuating electric field. The latter can disturb energy levels of a system. For comparison, spectral diffusion is also revealed in organic phosphors but is not observed in selforganizing QDs inserted into a matrix, because their local surroundings does not chance with the lapse of time. Thus, optical properties are dependent on type and amount of ligands connected with surface of nanocrystals. Indeed, these parameters should be controlled carefully at the stage of conjugation and solubilization in the process of QDs formation. In addition, ligands should be also carefully chosen, because their functional properties define behavior of NCs in solution. Modifiers of different 
type (polyethylene glycol, polysaccharose, polyvinyl alcohol etc.) or obtaining in aqueous solution are used in order to decrease the influence of environment on QD properties. The bifunctional molecules are used as stabilizers, for example, thioglycolic acid (TGA), 2mercaptoethylamine, cysteine. In this case, particles are stabilized not by formation of a structure mechanical barrier on the NC surface, but by charge on this surface, which prevents their approaching and further agglomeration $[7,8]$.

However, the current problem is complication in determining the exact concentration of NCs in colloid solutions. The methods used up to date require availability of expensive equipments, complicated mathematic calculations or plotting gauge dependences $[9,10]$. This is due to the obtained solutions consisting of NCs of various sizes. At long time storing, the process of aggregation, or decrease in NC sizes, occurs due to a transition of CdTe monomers to solution. This was confirmed by observation of a new maximum in the long-wave part of spectrum which is theoretically described by the classic nucleation theory. The energy of NCs consists of the volume energy, which is proportional to $R^{3}\left(G=-(4 / 3) \pi r^{3} \Delta G_{v}\right)$, and surface energy, which is proportional to $R^{2}\left(G=4 \pi \sigma r^{2}\right.$, where $\sigma$ is the surface tension). Thus, the critical size of NC is $r_{\mathrm{cr}}=2 \sigma V /(R T \ln S)$. Crystals with the size less than critical dissolve in solution and with the size higher than critical continue to grow.

Taking into account the aforesaid, the goal of this work is to study regularities in obtaining solutions of CdTe NCs containing particles of similar sizes and stabilized with thioglycolic acid by means of fractional precipitation from initial polydisperse solutions, and, subsequently, the investigation of photoluminescence (PL) properties of these systems.

\section{Experimental}

The reaction chamber with a mixture of substances controlling nucleation and growth of NCs was used to obtain colloid CdTe QDs. Before synthesis of QDs, necessary types of atoms forming NCs were loaded in the form of a precursor (the molecule or complex with one or more atoms required for growth of $\mathrm{NCs}$ ) into the reactor. Subsequently, precursors decompose and form new reaction units (monomers). These monomers cause nucleation and growth of NCs. A liquid in a reactor supplies energy required for decomposing precursors. On the other hand, this energy can be provided by means of thermal collision or chemical reaction between liquid medium and precursors, or due to combination of both mechanisms.

Intensely luminescent TGA-capped CdTe NCs in aqueous colloidal solutions were prepared using the procedure described in detail in the previous paper [3]. In a typical synthesis, $0.01 \mathrm{~mol}$ of $\mathrm{CdI}_{2}$ is dissolved in $170 \mathrm{ml}$ of water, and $0.03 \mathrm{~mol}$ of the thiol stabilizer TGA is added under stirring, followed by adjusting the
$\mathrm{pH}(\mathrm{pH}=11)$ to appropriate values by dropwise addition of $1 \mathrm{M}$ solution of $\mathrm{NaOH}$. The solution may be slightly turbid at this stage because of the insufficient solubility of Cd thiolate complexes, but this does not influence on the subsequent reactions. The reaction mixture is placed in a three-necked flask fitted with a septum and valves and is desaerated by Ar bubbling for $20 \mathrm{~min}$. Under stirring, $\mathrm{H}_{2} \mathrm{Te}$ is passed through the solution together with a slow nitrogen flow for $2 \mathrm{~min}$.

It was ascertained that the obtained solutions contain particles with sizes from 2 up to $10 \mathrm{~nm}$. The monodisperse QDs with a definite size can be obtained only by means of further fractionating of initial colloid solution.

To above-mentioned effect, $30 \mathrm{ml}$ (without using rotational evaporation) from $250 \mathrm{ml}$ of initial solution was used for the experimental investigation. Subsequently, $4.5 \mathrm{ml}$ was remained for the investigation of PL spectra and $25.5 \mathrm{ml}$ was used for fractional precipitation of $\mathrm{CdTe} \mathrm{NCs}$ by using the ion-selective method with isopropyl alcohol (IPA) [11].

The first bright orange fraction was extracted by addition of $5.69 \mathrm{ml}$ of IPA to initial solution for $15 \mathrm{~min}$ at constant stirring by using a magnetic stirrer and without heating. A solution with opalescence feature was centrifuged for $15 \mathrm{~min}$ with a rate of $500 \mathrm{~min}^{-1}$. Subsequently, the precipitate was peptized in $8.5 \mathrm{ml}$ of deionized water and $3.58 \mathrm{ml}$ of IPA for precipitation of CdTe NCs of less sizes was added into solution obtained after extraction of floc of the first fraction by decantation.

Precipitation of CdTe NCs of the second fraction was also carried out at constant stirring and weak heating for $15 \mathrm{~min}$. The bright yellow solution with opalescence feature was centrifuged for $20 \mathrm{~min}$ with the rate of $1000 \mathrm{~min}^{-1}$. The precipitate, visually more compact in comparison with that of the first fraction, was peptized in $8.5 \mathrm{ml}$ of deionized water.

$2.4 \mathrm{ml}$ of IPA was added up to opalescence into solution of the second fraction. Subsequently, the precipitation of the third fraction was carried out in the same manner as the second fraction. The bright yellow solution was centrifuged for $10 \mathrm{~min}$ with the rate of $1500 \mathrm{~min}^{-1}$. In this case, the floc was very little conglomeration of small size particles of bright orange CdTe NCs.

IPA was added up to opalescence into solution of the third fraction. But in this case, the precipitation of the fourth fraction was not observed by both increasing of isopropyl alcohol (additional $4 \mathrm{ml}$ after opalescence) and rates up to $8000 \mathrm{~min}^{-1}$.

Flocs of all the fraction peptized in deionized water were characterized by $\mathrm{pH}=5-6$. In this case, measurements of PL spectra were carried out immediately after precipitation.

The first fraction was segregated by repeated precipitation in order to clean the solution from excess stabilizer and products of reaction, and increasing the 
monodisperse degree. $26.5 \mathrm{ml}$ of IPA at constant stirring and weak heating for $15 \mathrm{~min}$ was used for reprecipitation. Contrary, without IPA, CdTe NCs was not precipitated increasing the rate even up to $10,000 \mathrm{~min}^{-1}$. The obtained solution was centrifuged for $10 \mathrm{~min}$ with the rate of $1000 \mathrm{~min}^{-1}$. After precipitation and separation by decantation, the bright orange precipitate was observed. Subsequently, the peptization by two means was carried out:

1) in deionized water with $0.8 \mathrm{ml}$ TGA per $100 \mathrm{ml}$ $\mathrm{H}_{2} \mathrm{O}$ for additional NCs stabilization by addition of $\mathrm{NaOH}$ to $\mathrm{pH}=8$;

2) in deionized water by addition of $\mathrm{NaOH}$ to $\mathrm{pH}=8$, but without TGA.

The precipitate of the second fraction was obtained by addition of $36 \mathrm{ml}$ of IPA with further centrifugation with the rate $1500 \mathrm{~min}^{-1}$ for $10 \mathrm{~min}$. The obtained precipitate was peptized similarly to previous case in deionized water and deionized water with $0.4 \mathrm{ml}$ TGA in $100 \mathrm{ml} \mathrm{H}_{2} \mathrm{O}$. The third fraction was obtained by addition of $16 \mathrm{ml}$ of IPA and, subsequently, the solution was centrifugated with the rate $2000 \mathrm{~min}^{-1}$ for $10 \mathrm{~min}$. The floc was divided into three parts and peptized by a three means:

1) in deionized water;

2) in solution with $0.3 \mathrm{ml}$ of TGA per $100 \mathrm{ml} \mathrm{H}_{2} \mathrm{O}$;

3 ) in solution of sodium polyphosphate.

The precipitate of the fourth fraction was peptized in deionized water with controlling $\mathrm{pH}$ by $\mathrm{NaOH}$ and further extracted by chloroform.

The photoluminescence spectra were measured at room temperature.

\section{Results and discussion}

The formation process of the CdTe NCs can be identified from both the color change and the PL spectra of the solution. As the reaction proceeded, the solution was observed to change from colorless to transparent yellow to light brown and then red brown. This directly indicated the formation of differently-sized CdTe NCs. Carrying out the reactions in the presence of thiols (RSH) results in a surface modification of the NCs and reduction of the non-radiative local surface traps, which in turn, leads to an enhancement of the quantum yield of the excitonic transitions.

Shown in Fig. 1 is the change of PL spectra of a CdTe QDs in the process of precipitation of monodisperse fractions. Thus, the spectra of initial solution and all of fractions have relatively narrow band of excitonic luminescence with some shift to the longwave part. Spectra of initial solution and the first two fractions of a CdTe QDs are characterized by the peak wavelengths $580 \mathrm{~nm}$ (Fig. 1, curves A, 1, 2), and spectra of the third fraction by $575 \mathrm{~nm}$ (Fig. 1, curve 3). Analysis of PL spectra shows that precipitation of the first two fractions does not activate the shift of maximum in PL spectra that correspond to excitonic recombination, but in this case, the splitting of peak in the PL spectrum is observed. The latter probably is caused by recombination through surface levels related with defects. PL intensities of the first and second fractions of CdTe QDs are significantly higher than the corresponding intensity for initial solution (approximately twice higher for the first fraction). The third fraction is characterized by insignificant shift of maximum into the short-wave region, decreasing intensity and widening the spectral line.

The re-precipitation of the first fraction results in insignificant narrowing the spectral lines and their shift into the long-wave region. In either of the obtained spectra, the splitting of peak was not observed. The most intensive PL of CdTe QDs (almost four times larger than the corresponding amount for initial solution) is observed in the process of re-precipitation of the first fraction. Comparison of PL spectra of re-precipitated first fraction allows concluding that additional content of stabilizer results in a significant decrease in their intensity (Fig. 2, curves 1a, 1b). Correspondingly, PL spectra of the second fraction allow concluding that too little stabilizer content results in the same effect. Since the next fraction contains stabilizer in less amount than the previous fraction, the stabilizer should be added in order to avoid any decrease in the PL intensity of a system, starting from the second fraction in the further process of re-precipitation (Fig. 2, curves 2a, 2b, 3a, 3b).

The PL intensity of the next fraction decreases in comparison with the previous one. Indeed, this effect is due to the CdTe QD concentration that is highest in the first fraction. This concentration decreases in the process of further fractionating. The latter is confirmed by the fact that in all the prepared fractions, only the first fraction can be segregated repeatedly, as well as the PL intensity decreases in the process of further precipitation of the first fraction. Thus, in the fourth fraction, the CdTe QD concentration is too small, and subsequently

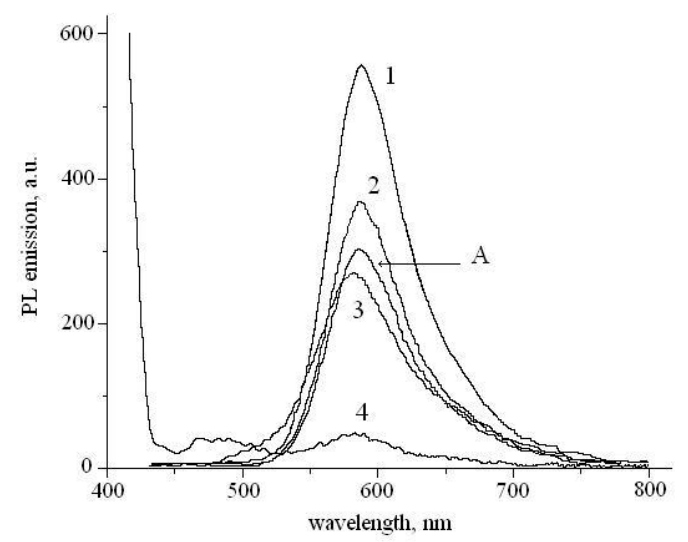

Fig. 1. The PL spectra (293 K) of TGA-capped CdTe NCs in aqueous colloidal solutions (A) and PL spectra of sizeselected fractions isolated of a crude solution of CdTe for four fractions (1-4). 


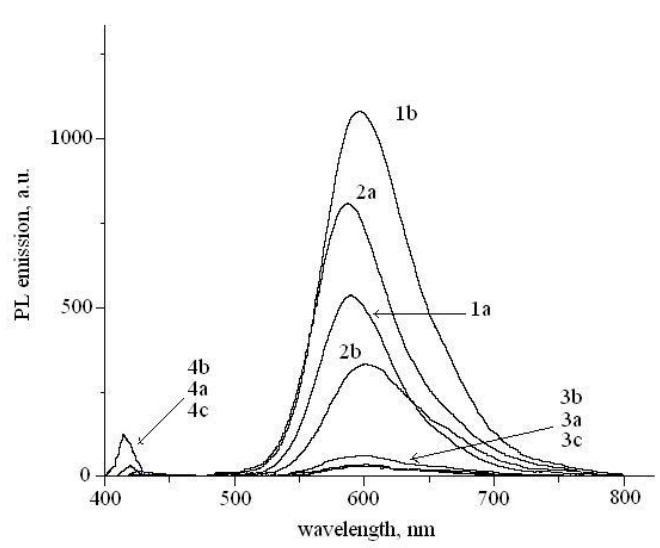

Fig. 2. The PL spectra (293 K) of re-deposited first fraction CdTe NCs with the next flocculus resolution using the TGA solution (1a, 2a, 3a, 4a), deionized water (1b, 2b, 3b, 4b), sodium phosphate solution (3c) and chloroform (4 c) (1-4 indicate the fraction number).

PL disappears almost completely (Fig. 2, curves $4 a-4 c)$. Also the stability of the obtained solutions is too low.

\section{Conclusions}

Technology of manufacturing the water-soluble CdTe QDs has been reported in this paper. Functional groups of the thiol capping molecules on the nanocrystals provide their water solubility, high processability and a desired surface charge. The method reported here is also very attractive owing to its simplicity as compared to other methods for producing water-soluble semiconductor QDs. It was ascertained that precipitation allows obtaining the CdTe QDs with significantly less size scatter than in the initial solution, and also cleaning them from an excess stabilizer and reaction products. Re-precipitation of the first fraction allows to separate CdTe QDs characterized with the highest PL intensity (approximately four times higher than the intensity of initial solution). Because of colloid NCs are dispersed in solution, they are not related with any solid phase. Thus, CdTe QDs can be obtained commercially in a reaction chamber and then deposited on suitable substrate or inserted into a matrix. The obtained results can find an applied interest in development of new composite materials based on CdTe as well as in making quickresponse effective optoelectronic devices for nanoelectronics.

\section{References}

1. T. Koyama, S. Ohtsuka, H. Nagata and S. Tanaka, Fabrication of microcrystallites of II-IV compound semiconductors by laser ablation method // $J$. .Cryst. Growth, 117 (1-4), p. 156-160 (1992).

2. J. Hia, P. Yang, Y. Sun, One-dimentional nanostructures: synthesis, characterization, and applications // Adv. Mater., 15 (5), p. 353-389 (2003).

3. R. He, X. You, H. Tian, F. Gao, D. Cui, Synthesis of CdTe colloidal quantum dots (QDs) in water // Front. Chem. China, 3(3), p. 325-329 (2008).

4. A.D. Yoffe, Low-dimensional systems: quantum size effects and electronic properties of semiconductor microcrystallites (zero-dimensional systems) and some quasi-two-dimensional systems // Adv. Phys., 42, p. 173-262 (1993).

5. A.L. Rogach, Nanocrystalline CdTe and $\mathrm{CdTe}(\mathrm{S})$ particles: wet chemical preparation, size-dependent optical properties and perspectives of optoelectronic applications // Mater. Sci. Eng. B, 69, p. 435-440 (2000).

6. T. Rajh, O.I. Micic, A.J. Nosik, Synthesis and characterization of surface-modified colloidal cadmium telluride quantum dots // J. Phys. Chem., 97(46), p. 11999-12003 (1993).

7. N. Gaponik, D.V. Talapin, A.L. Rogach, K. Hoppe, E.V. Shevchenko, A. Kornowski, A. Eychmuller, H. Weller, Thiol-capping of CdTe nanocrystals: an alternative to organometallic synthetic routes // $J$. Phys. Chem. B, 106, p. 7177-7185 (2002).

8. W.W. Yu, L. Qu, W. Guo, X. Peng, Experimental determination of the extinction coefficient of CdTe, CdSe, and CdS nanocrystals // Chem. Mater., 15, p. 2854-2860 (2003).

9. A.R. Bustos, J.R. Encinar, M.T. FernándezArgüelles, J.M. Costa-Fernández, A. Sanz-Medel, Elemental mass spectrometry: a powerful tool for an accurate characterisation at elemental level of quantum dots // Chem. Communs., p. 3107-3109 (2009).

10. J. Sun, E.M. Goldys, Linear absorption and molar extinction coefficients in direct semiconductor quantum dots // J. Phys. Chem. C, 112 (25), p. 9261-9266 (2008).

11. Yu.B. Khalavka, P.I. Feychuk, L.P. Shcherbak, Dimension-selective fractionating the CdTe colloidal particles by using the stabilized thiols // Naukovyi visnyk Chernivets. Universitetu, No.270, Khimiya, p. 18-24 (2005), in Ukrainian. 